\title{
Interaction-free evolving states of a bipartite system
}

\author{
A. Napoli ${ }^{1}$ M. Guccione, ${ }^{1}$ A. Messina, ${ }^{1}$ and D. Chruściński ${ }^{2}$ \\ ${ }^{1}$ Dipartimento di Fisica e Chimica, Palermo University, via Archirafi 36, 90123 Palermo, Italy \\ ${ }^{2}$ Institute of Physics, Faculty of Physics, Astronomy and Informatics Nicolaus Copernicus University, Grudziadzka 5/7, 87-100 Torun, Poland
}

(Received 25 February 2014; published 5 June 2014)

\begin{abstract}
We show that two interacting physical systems may admit entangled pure or nonseparable mixed states evolving in time as if the mutual interaction Hamiltonian were absent. In this paper we define these interaction-free evolving (IFE) states and characterize their existence for a generic binary system described by a time-independent Hamiltonian. A comparison between IFE subspace and the decoherence-free subspace is reported. The set of all pure IFE states is explicitly constructed for a nonhomogeneous spin-star-system model
\end{abstract}

DOI: 10.1103/PhysRevA.89.062104

PACS number(s): 03.65.-w

\section{INTRODUCTION}

Consider a bipartite system $S$ consisting of two quantum interacting subsystems $A$ and $B$ with free Hamiltonians $H_{A}$ acting on the Hilbert space $\mathcal{H}_{A}$ and $H_{B}$ acting on $\mathcal{H}_{B}$, respectively. The states of $A+B$ live in the Hilbert space $\mathcal{H}=\mathcal{H}_{A} \otimes \mathcal{H}_{B}$, where the Hamiltonian of the bipartite system is

$$
H=H_{A}+H_{B}+H_{I}=H_{0}+H_{I},
$$

with $H_{I}$ being the operator describing the coupling between $A$ and $B$. Generally speaking, the entanglement exhibited in the initial pure or mixed state of the bipartite system, regardless of how it is measured, undergoes changes over time traceable back to the presence of $H_{I}$ in the Hamiltonian. Thus, for example, an initial factorized pure state or a separable mixed state evolves into an entangled state where time-dependent classical and/or quantum correlations between $A$ and $B$ generally emerge. In such a general dynamical scenario the increasing attention to the existence in some bipartite systems of subradiant states that are selected pure factorized states which evolve, keeping the system in its fully initial decorrelated condition at any time instant, is not surprising. Such peculiar behavior, of both fundamental [1,2] and applicative interest [3-8], results from quantum interference effects canceling in the evolved state, at a generic time instant, exactly those contributions, stemming from the superposition principle, which, otherwise, would determine the onset and possibly the persistence of correlation manifestations between $A$ and $B$. Subradiance is a cooperative effect that has been investigated both theoretically $[1,2,9-26]$ and experimentally [4,27-32] following the seminal paper by Dicke [1], mainly in radiation-matter systems, where it describes optically inactive states of an atomic ensemble $(A)$ in an electromagnetic environment $(B)$. The current upsurge of interest in these states reflects, indeed, the existence of many other physical contexts where this phenomenon may find promising applications $[4,33-35]$ as well as the experimental evidence that a system made up of superconducting qubits or a diatomic molecule in an optical lattice may be prepared in subradiant states. In connection with such an enlarged view we appropriately recall that the name "subradiant states" has also been adopted [36] to classify factorized states of generic bipartite systems from which the two subsystems evolve with no energy exchange between them, maintaining moreover their statistical independence. In this paper we call the subradiant state a generalized state of this type, regardless of the specific nature of both subsystems.

Recently, for instance, the environmental noise plaguing the unitary evolution of superconducting artificial atoms in a circuit QED setting has been modeled by coupling the dynamical variables of the circuit to the degrees of freedom of a fermionic bath. Systems of this type, where bosonic degrees of freedom are absent, might admit subradiant states under appropriate conditions [37].

In this paper we go beyond the original notion of subradiance investigating the existence of initially entangled pure or mixed states of the bipartite system evolving as if $A$ and $B$ were decoupled. This condition, guaranteeing the absence of energy exchanges between the two subsystems, might be of interest in any applicative protocol based on quantum processes involving storage steps. Another dynamical property of such states is that the quantum covariance of any pair of observables $\mathcal{O}_{A}$ and $\mathcal{O}_{B}$ acting on $\mathcal{H}_{A}$ and $\mathcal{H}_{B}$, each one invariant with respect to the free evolution of the corresponding subsystem, keeps its initial value even if such observables do not commute with $H_{I}$. When states of this kind exist, we call them interaction-free evolving (IFE) states of the bipartite system. These states should not be confused with decoherence-free states giving rise to the celebrated decoherence-free subspaces (DFS; see, e.g., the review in [38]). DFS are analyzed in the context of nonunitary evolution of an open quantum system living in some Hilbert space $\mathcal{H}$. One says that a linear subspace $\widetilde{\mathcal{H}} \subset \mathcal{H}$ provides a DFS if the evolution of the system restricted to $\widetilde{\mathcal{H}}$ is unitary. Under appropriate conditions $[39,40]$ if the initial state vector belongs to $\widetilde{\mathcal{H}}$, it stays there and hence does not lose quantum coherence. Here, we assume that the evolution of the bipartite system is unitary on $\mathcal{H}_{A} \otimes \mathcal{H}_{B}$. Of course, subsystem $B$ might be able to play the role of environment of $A$. We emphasize that in this case too an IFE state is a state of the compound system $A+B$, unitarily evolving on $\mathcal{H}_{A} \otimes \mathcal{H}_{B}$. Generally speaking, as previously underlined, in an IFE state $A$ and $B$ exhibit entanglement at all times even though it might also happen that an IFE state keeps the factorized form $\left|\psi_{A}(t)\right\rangle\left|\psi_{B}(t)\right\rangle$ as time progresses. In this case state $\left|\psi_{A}(t)\right\rangle$, belonging to $\mathcal{H}_{A}$, is indeed a decoherencefree state since $\left|\psi_{A}(t)\right\rangle=\exp \left(-i H_{A} t\right)\left|\psi_{A}(0)\right\rangle$ by the IFE state definition. Recall that if we consider a nonunitary evolution as a reduction of the unitary one when the system $S$ is coupled 
to an environment $E$ and the interaction system-environment Hamiltonian reads $H_{S E}=\sum_{\alpha} S_{\alpha} \otimes E_{\alpha}$, then DFS is spanned by vectors $|\psi\rangle$ satisfying $S_{\alpha}|\psi\rangle=\lambda_{\alpha}|\psi\rangle$ [38]. Hence, if all system operators $S_{\alpha}$ are Hermitian, then a nontrivial DFS $\widetilde{\mathcal{H}}$ exists only when all $S_{\alpha}$ mutually commute on $\tilde{\mathcal{H}}$. Interestingly, as we show in this paper, a similar condition governs the existence of IFE states.

The main result of this paper is the construction of the characteristic equation for both pure and mixed IFE states, that is, the equation whose set of solutions singles out all and only the IFE states of a given bipartite system. In order to demonstrate the practical usefulness of such an equation, we solve it in the nontrivial case of a nonhomogeneous spin-star system, finding all its IFE pure states.

\section{IFE PURE STATES}

Let us consider the following.

Definition 1. A normalized vector $|\psi\rangle \in \mathcal{H}$ is an IFE pure state if it satisfies the following equation:

$$
e^{-i H t}|\psi\rangle \sim e^{-i H_{0} t}|\psi\rangle
$$

where the approximate symbol $(\sim)$ denotes an equivalence relation: $|\psi\rangle \sim|\phi\rangle$ iff $|\psi\rangle=e^{i \alpha}|\phi\rangle$, with $\alpha$ being a real number (a relative phase).

This means that $|\psi\rangle$ is an IFE state iff there exists a real function of time $\alpha(t)$ such that

$$
e^{-i H t}|\psi\rangle=e^{-i \alpha(t)} e^{-i H_{0} t}|\psi\rangle
$$

at any time instant $t$. It is easy to convince oneself that this definition necessarily implies that $\alpha(t)$ is linear in $t$, i.e., $\alpha(t)=$ $\alpha_{0}+\alpha t$. We prove this statement in the Appendix [41]. Since the global phase $\alpha_{0}$ does not have any physical meaning, we take $\alpha_{0}=0$. We thus substitute Eq. (3) with the following equation:

$$
e^{-i H t}|\psi\rangle=e^{-i \alpha t} e^{-i H_{0} t}|\psi\rangle
$$

In order to characterize all the IFE pure states of the system, let us begin by stating that $|\psi\rangle$ is a solution of Eq. (3) iff, for any nonnegative integer $n$,

$$
H^{n}|\psi\rangle=\left(H_{0}+\alpha \mathbb{I}\right)^{n}|\psi\rangle,
$$

which implies that Eq. (3) is satisfied for all $t$. For $n=1$ one obtains

$$
H_{I}|\psi\rangle=\alpha|\psi\rangle
$$

that is, $|\psi\rangle$ defines an eigenvector of $H_{I}$ and $\alpha$ denotes the corresponding eigenvalue. This means that $|\psi\rangle$ is a zero mode of $H_{I}^{(\alpha)}:=H_{I}-\alpha \mathbb{I}$, i.e.,

$$
|\psi\rangle \in \operatorname{Ker} H_{I}^{(\alpha)} .
$$

Moreover, starting from Eq. (5) and exploiting Eq. (7), we also obtain

$$
H_{I}^{(\alpha)} H_{0}|\psi\rangle=0
$$

and, by induction,

$$
H_{I}^{(\alpha)} H_{0}^{n}|\psi\rangle=0
$$

for all $n$. Now, for any eigenvalue $\alpha$ of $H_{I}$, let us define

$$
\mathcal{N}_{\alpha}:=\bigcap_{n} \operatorname{Ker}\left(H_{I}^{(\alpha)} H_{0}^{n}\right) .
$$

It is clear that $\mathcal{N}_{\alpha}$ defines a linear subspace of $\mathcal{H}$. Of course, it may happen that $\mathcal{N}_{\alpha}=\{0\}$. It is easy to show that if $|\psi\rangle \in$ $\mathcal{N}_{\alpha} \neq\{0\}$, then Eq. (3) holds. In this way we have proved the following theorem:

Theorem 1. A vector $|\psi\rangle \in \mathcal{H}$ is an IFE state iff $|\psi\rangle \in$ $\mathcal{N}_{\alpha} \neq\{0\}$ for some eigenvalue $\alpha$ of the interaction part $H_{I}$.

It is clear that the space $\mathcal{N}$ of IFE states is stratified into mutually orthogonal sectors

$$
\mathcal{N}=\bigcup_{\alpha} \mathcal{N}_{\alpha},
$$

with $\mathcal{N}_{\alpha} \perp \mathcal{N}_{\beta}$ for $\alpha \neq \beta$. In particular, if $|\psi\rangle \in \mathcal{N}_{0}$, then

$$
e^{-i H t}|\psi\rangle=e^{-i H_{0} t}|\psi\rangle
$$

at any time instant $t$.

Now, we show that formula (10) defining $\mathcal{N}_{\alpha}$ may be considerably simplified. Note that

$$
\left.\left[H_{0}, H_{I}\right]\right|_{\mathcal{N}_{0}}=0 .
$$

Indeed, for any $|\psi\rangle \in \mathcal{N}_{0}$ one finds $H_{0} H_{I}|\psi\rangle-H_{I} H_{0}|\psi\rangle=$ 0 . Conversely, if $|\psi\rangle \in \operatorname{Ker} H_{I}$ and $\left[H_{0}, H_{I}\right]|\psi\rangle=0$, then $H_{I} H_{0}^{n}|\psi\rangle=0$ for $n=1,2, \ldots$. To prove this let $\mathcal{M}=$ $\operatorname{Ker}\left[H_{0}, H_{I}\right]$ and let $\left\{\left|e_{1}\right\rangle, \ldots,\left|e_{r}\right\rangle\right\}$ be an orthonormal basis in $\mathcal{M}$ such that

$$
\left.H_{0}\right|_{\mathcal{M}}=\sum_{k=1}^{r} a_{k}\left|e_{k}\right\rangle\left\langle e_{k}\right|
$$

and

$$
\left.H_{I}\right|_{\mathcal{M}}=\sum_{k=1}^{r} b_{k}\left|e_{k}\right\rangle\left\langle e_{k}\right|
$$

provide spectral decompositions of $H_{0}$ and $H_{I}$ restricted to $\mathcal{M}$. Now, let $|\psi\rangle \in \operatorname{Ker} H_{I}$ and $|\psi\rangle \in \operatorname{Ker}\left[H_{0}, H_{I}\right]$; that is, we assume that $\operatorname{Ker} H_{I} \cap \mathcal{M} \neq\{0\}$. Suppose that $\operatorname{Ker} H_{I} \cap \mathcal{M}$ is spanned by $\left\{\left|e_{1}\right\rangle, \ldots,\left|e_{l}\right\rangle\right\}$ with $l \leqslant r$, that is, $\left.H_{I}\right|_{\mathcal{M}}=$ $\sum_{k=l+1}^{r} b_{k}\left|e_{k}\right\rangle\left\langle e_{k}\right|$ due to $H_{I}\left|e_{k}\right\rangle=0$ for $k=1, \ldots, l$. One immediately finds

$$
H_{I} H_{0}^{n}|\psi\rangle=\sum_{k=l+1}^{r} a_{k}^{n} b_{k}\left|e_{k}\right\rangle\left\langle e_{k} \mid \psi\right\rangle=0
$$

due to the fact that $|\psi\rangle=\sum_{k=1}^{l} x_{k}\left|e_{k}\right\rangle \in \operatorname{Ker} H_{I} \cap \mathcal{M}$. Hence, $H_{I} H_{0}^{n}|\psi\rangle=0$ whenever $H_{I}|\psi\rangle=0$ and $\left[H_{0}, H_{I}\right]|\psi\rangle=0$. In a similar way one shows that $H_{I}^{(\alpha)} H_{0}^{n}|\psi\rangle=0$ whenever $H_{I}^{(\alpha)}|\psi\rangle=0$ and $\left[H_{0}, H_{I}^{(\alpha)}\right]|\psi\rangle=0$.

Corollary 1. The subspace $\mathcal{N}_{0}$ may be represented as follows:

$$
\mathcal{N}_{0}=\operatorname{Ker} H_{I} \cap \operatorname{Ker}\left[H_{0}, H_{I}\right],
$$

and similarly,

$$
\mathcal{N}_{\alpha}=\operatorname{Ker} H_{I}^{(\alpha)} \cap \operatorname{Ker}\left[H_{0}, H_{I}^{(\alpha)}\right]
$$

for any eigenvalue $\alpha$ of the interaction part $H_{I}$. 
It is clear that to define $\mathcal{N}_{\alpha}$ one has to solve eigenvalues of $H_{I}$ which might be highly nontrivial. One may ask a simpler question, namely, how to check whether IFE states do exist. Combining (17) and (18), one arrives at the following existence condition:

Corollary 2. A Hamiltonian $H=H_{0}+H_{I}$ allows for IFE states if and only if $\operatorname{Ker}\left[H_{0}, H_{I}\right]$ is nontrivial.

Indeed, if $|\psi\rangle$ is an IFE state, then there exists $\bar{\alpha} \in \mathbb{R}$, an eigenstate of $H_{I}$, such that $\mathcal{N}_{\bar{\alpha}}$ is not trivial. This existence in turn implies that $|\psi\rangle \in \operatorname{Ker}\left[H_{0}, H_{I}^{\bar{\alpha}}\right]=\operatorname{Ker}\left[H_{0}, H_{I}\right]$. Vice versa, if $\mathcal{M}=\operatorname{Ker}\left[H_{0}, H_{I}\right]$ is not trivial, $H_{0}$ and $H_{I}$ may be simultaneously diagonalized in $\mathcal{M}$, and each common eigenstate is an IFE state since it belongs to $\mathcal{N}_{\alpha}$ for some $\alpha$. We emphasize that had we put $\alpha=0$ in Eq. (3), the existence of IFE states belonging to the restricted set accordingly defined would not be guaranteed by the condition expressed by Corollary 2. The reason is that we cannot be sure to find zero among the eigenvalues of $H_{I}$ restricted to $\mathcal{M}$.

Suppose now that one deals with a bipartite system in $\mathcal{H}=$ $\mathcal{H}_{A} \otimes \mathcal{H}_{B}$ described by

$$
H_{0}=H_{A}+H_{B}
$$

and the interaction term $H_{I}$ (to simplify notation we identify $H_{A}$ with $H_{A} \otimes \mathbb{I}_{B}$ and similarly for $H_{B}$ ). Note that the corresponding bipartite IFE states do exhibit the absence of energy exchanges between subsystems $A$ and $B$. Indeed, for any $t$ one finds

$$
\begin{aligned}
\mathcal{E}_{A}(t) & :=\left\langle\psi\left|e^{i H t} H_{A} e^{-i H t}\right| \psi\right\rangle \\
& =\left\langle\psi\left|e^{i H_{0} t} H_{A} e^{-i H_{0} t}\right| \psi\right\rangle=\left\langle\psi\left|H_{A}\right| \psi\right\rangle
\end{aligned}
$$

and

$$
\begin{aligned}
\mathcal{E}_{B}(t) & :=\left\langle\psi\left|e^{i H t} H_{B} e^{-i H t}\right| \psi\right\rangle \\
& =\left\langle\psi\left|e^{i H_{0} t} H_{B} e^{-i H_{0} t}\right| \psi\right\rangle=\left\langle\psi\left|H_{B}\right| \psi\right\rangle,
\end{aligned}
$$

which shows that energies $\mathcal{E}_{A}(t)$ and $\mathcal{E}_{B}(t)$ of the two subsystems are conserved. Of course, the converse is generally not true. Let us consider, for example, the time evolution obtained starting from a stationary state of $H$. Under this condition the mean values of both $H_{A}$ and $H_{B}$, as well as of any time-independent observable of the system, are obviously stationary, but the eigenstates of $H$ do not, in general, satisfy Eq. (3).

\section{IFE MIXED STATES}

In this section we generalize the notion of IFE for mixed states. Denote by $\mathcal{S}(\mathcal{H})$ the space of density operators living in $\mathcal{H}$ and consider the Hamiltonian dynamics generated by (1). One has the following generalization of Definition 1.

Definition 2. A density operator $\rho \in \mathcal{S}(\mathcal{H})$ is an IFE mixed state if it satisfies the following equation:

$$
e^{-i H t} \rho e^{i H t}=e^{-i H_{0} t} \rho e^{i H_{0} t}
$$

at any time instant $t \in \mathbb{R}$.

It is clear that if $\rho=|\psi\rangle\langle\psi|$, then the above definition reproduces Definition 1 .

Let $\left|\psi_{\alpha}^{i}\right\rangle$ denotes an orthonormal basis in $\mathcal{N}_{\alpha}$, that is,

$$
H_{I}\left|\psi_{\alpha}^{i}\right\rangle=\alpha\left|\psi_{\alpha}^{i}\right\rangle
$$

for $i=1, \ldots, n_{\alpha}=\operatorname{dim} \mathcal{N}_{\alpha}$. One immediately has the following corollary.

Corollary 3. A density operators $\rho$ defines an IFE mixed state iff

$$
\rho=\sum_{\alpha} \sum_{i, j=1}^{n_{\alpha}} p_{\alpha}^{(i, j)}\left|\psi_{\alpha}^{i}\right\rangle\left\langle\psi_{\alpha}^{j}\right|,
$$

where $p_{\alpha}^{(i, j)} \geqslant 0$ and $\sum_{\alpha} \sum_{i, j=1}^{n_{\alpha}} p_{\alpha}^{(i, j)}=1$.

Let us observe that any IFE mixed state defines a direct sum of positive operators

$$
\rho=\bigoplus_{\alpha} \rho_{\alpha},
$$

where

$$
\rho_{\alpha}=\sum_{i, j=1}^{n_{\alpha}} p_{\alpha}^{(i, j)}\left|\psi_{\alpha}^{i}\right\rangle\left\langle\psi_{\alpha}^{j}\right|
$$

is supported on $\mathcal{N}_{\alpha}$. Hence any IFE pure state belongs to single sector $\mathcal{N}_{\alpha}$, whereas a genuine IFE mixed state defines a mixture of positive operators supported on all sectors $\mathcal{N}_{\alpha}$.

Again, it is clear that if one deals with a bipartite system and if $\rho_{A B}$ is an IFE state, then

$$
\mathcal{E}_{A}(t)=\operatorname{Tr}\left(e^{-i H t} \rho_{A B} e^{i H t} H_{A}\right)=\operatorname{Tr}\left(\rho_{A B} H_{A}\right),
$$

and the same is true for $\mathcal{E}_{B}(t)$. Hence there is no energy exchange between subsystems $A$ and $B$ for any IFE mixed state.

\section{IFE PURE STATES OF A NONHOMOGENEOUS SPIN-STAR SYSTEM}

Consider a nonhomogeneous spin-star system consisting of a central spin coupled to $N$ mutually not interacting spins around it. The Hamiltonian describing such a system has the form of (1) with

$$
H_{0}=\omega_{0} \sigma_{z}+\omega \sum_{i=1}^{N} \sigma_{z}^{(i)}
$$

and

$$
H_{I}=\sum_{i=1}^{N} \gamma_{i}\left(\sigma_{+} \sigma_{-}^{(i)}+\sigma_{-} \sigma_{+}^{(i)}\right) .
$$

The dynamical variables of the central spin are represented by the Pauli operators $\sigma_{z}, \sigma_{ \pm} \equiv \frac{1}{2}\left(\sigma_{x} \pm i \sigma_{y}\right)$, whereas the Pauli operators describing the $i$ th $(i=1, \ldots, N)$ spin are denoted by $\sigma_{z}^{(i)}, \sigma_{ \pm}^{(i)} \equiv \frac{1}{2}\left(\sigma_{x}^{(i)} \pm i \sigma_{y}^{(i)}\right)$.

Considering this physical system as bipartite and the central spin as one of the two subsystems, the main aim of this section is the construction of the set of all IFE pure states associated with the spin-star system under scrutiny. In order to do this let us begin by observing that a normalized state of our bipartite system can always be written in the form $|\Psi\rangle=|-\rangle\left|\psi_{-}\right\rangle+$ $|+\rangle\left|\psi_{+}\right\rangle$, where $| \pm\rangle$are the eigenstates of $\sigma_{z}$ with eigenvalues +1 and -1 , respectively, whereas $\left|\psi_{ \pm}\right\rangle$belong to the Hilbert space of the system constituted by the spins $1, \ldots, N$ and satisfying the condition $\left|\psi_{+}\right|^{2}+\left|\psi_{-}\right|^{2}=1$. 
In view of Corollaries 1 and 2, we must diagonalize $H_{0}$ and $H_{I}$ within the vectorial space $\operatorname{Ker}\left[H_{0}, H_{I}\right]$ provided $\operatorname{dim}\left(\operatorname{Ker}\left[H_{0}, H_{I}\right]\right)>0$. It is easy to demonstrate that the equation $\left[H_{0}, H_{I}\right]|\psi\rangle=0$ may be rewritten as follows:

$$
\begin{aligned}
& {\left[H_{0}, H_{I}\right]|\psi\rangle} \\
& \quad=2\left(\omega_{0}-\omega\right)\left[|+\rangle \sum_{i=1}^{N} \gamma_{i} \sigma_{-}^{(i)}\left|\psi_{-}\right\rangle-|-\rangle \sum_{i=1}^{N} \gamma_{i} \sigma_{+}^{(i)}\left|\psi_{+}\right\rangle\right]=0,
\end{aligned}
$$

which in turn requires the existence of solutions for the two equations

$$
\sum_{i=1}^{N} \gamma_{i} \sigma_{ \pm}^{(i)}\left|\psi_{ \pm}\right\rangle=0
$$

We solve Eq. (29), exploiting the method reported in Ref. [18]: let us introduce the operators $A_{ \pm}$given by

$$
A_{ \pm}=\exp \left(\sum_{i=1}^{N} g_{ \pm}^{(i)} \sigma_{z}^{(i)}\right)
$$

where the complex parameters $g_{ \pm}^{(i)}$ will be chosen later.

The two operators $A_{+}$and $A_{-}$thus defined are, in general, neither unitary nor Hermitian. However, they are not singular, and thus $A_{ \pm}^{-1}$ exist. Accordingly, Eq. (29) may be transformed as follows:

$$
A_{ \pm}^{-1} \sum_{i=1}^{2} \gamma_{i} \sigma_{ \pm}^{(i)} A_{ \pm} A_{ \pm}^{-1}\left|\psi_{ \pm}\right\rangle=0
$$

On the other hand, it is easy to demonstrate that

$$
A_{ \pm}^{-1} \sigma_{ \pm}^{(i)} A_{ \pm}=\sigma_{ \pm}^{(i)} e^{\mp 2 g_{ \pm}^{(i)}}
$$

and then, choosing the parameters $g_{ \pm}^{(i)}(i=1, \ldots, N)$ in such a way that $\gamma_{i}=\gamma e^{ \pm 2 g_{ \pm}^{(i)}}$ with $\gamma=\sqrt{\sum_{i=1}^{N} \gamma_{i}^{2}}$, the condition under which the state $|\Psi\rangle=|-\rangle\left|\psi_{-}\right\rangle+|+\rangle\left|\psi_{+}\right\rangle$belongs to the kernel of $\left[H_{0}, H_{I}\right]$ becomes

$$
\sum_{i=1}^{N} \gamma \sigma_{+}^{(i)}\left(A_{+}^{-1}\left|\psi_{+}\right\rangle\right)=0, \quad \sum_{i=1}^{N} \gamma \sigma_{-}^{(i)}\left(A_{-}^{-1}\left|\psi_{-}\right\rangle\right)=0 .
$$

These equations show that due to the operators $A_{ \pm}$we get rid of the nonhomogeneous character of Eq. (31) where it appears through the $i$ dependence of the coupling constants $\left(\gamma_{i}\right)$.

Let us note that the choice of the parameters $g_{ \pm}^{(i)}$ guarantees that the two operators $A_{+}$and $A_{-}$satisfy $A_{+} A_{-}=A_{-} A_{+}=$ I. Let's moreover observe that the states $\left|\widetilde{\psi}_{ \pm}\right\rangle=A_{ \pm}^{-1}\left|\psi_{ \pm}\right\rangle$ satisfying Eq. (33) are well known in terms of the simultaneous eigenstates $|r, m, v\rangle$ of the square and of the $z$ component of the total angular momentum of the $N$ uncoupled spins,

$$
S^{2}|r, m, v\rangle \equiv \frac{1}{2}\left(\sum_{i=1}^{N} \vec{\sigma}^{(i)}\right)^{2}|r, m, v\rangle=r(r+1)|r, m, v\rangle,
$$

where $r=0,1, \ldots, \frac{N}{2}$ if $N$ is even and $r=\frac{1}{2}, \frac{3}{2}, \ldots, \frac{N}{2}$ if $N$ is odd. Moreover

$$
S_{z}|r, m, v\rangle \equiv \frac{1}{2} \sum_{i=1}^{N} \sigma_{z}^{(i)}|r, m, v\rangle=m|r, m, v\rangle,
$$

with $m=-r-r+1, \ldots, r$. The quantum number $v=$ $1,2, \ldots, v(r)$, with

$$
v(r)=\left(\begin{array}{c}
N \\
\frac{N}{2}-r
\end{array}\right)+\left(\begin{array}{c}
N \\
\frac{N}{2}-r-1
\end{array}\right)
$$

and $\left(\begin{array}{c}N \\ -1\end{array}\right)=0$, allows us to distinguish between different states of the coupled angular momentum basis characterized by the same $r$ and $m$. It is possible to convince oneself that $\left|\widetilde{\psi}_{+}\right\rangle \equiv \sum_{r, v} C_{r, v}^{+}|r, r, v\rangle$ and $\left|\widetilde{\psi}_{-}\right\rangle \equiv \sum_{r, v} C_{r, v}^{-}|r,-r, v\rangle$, with $C_{r, v}^{ \pm} \in \mathbb{C}$. We may thus claim that a generic state $|\psi\rangle$ satisfying Eq. (28) may be written as follows:

$$
|\psi\rangle=|+\rangle \sum_{r, v} C_{r, v}^{+} A_{+}|r, r, v\rangle+|-\rangle \sum_{r, v} C_{r, v}^{-} A_{-}|r,-r, v\rangle .
$$

It is remarkable that $\operatorname{Ker}\left[H_{0}, H_{I}\right]$ for the Hamiltonian model under scrutiny coincides with $\operatorname{Ker} H_{I}$, which means that $H_{I}|\psi\rangle=0$ iff $|\psi\rangle$ is given by Eq. (37). This result is a direct consequence of the fact that the resolution of the equation $H_{I}|\psi\rangle=0$ leads exactly to Eqs. (33). In view of Corollary 2 we may thus claim that $\mathcal{N}_{\alpha}$ is empty for each eigenvalue $\alpha \neq 0$ of $H_{I}$. We thus may conclude that the space $\mathcal{N}$ of IFE pure states for our Hamiltonian model coincides with $\mathcal{N}_{0}$. It is interesting to investigate the diagonalization problem of $H_{0}$ within $\mathcal{N} \equiv \mathcal{N}_{0}$. To this end let's observe that both the operators $A_{+}$and $A_{-}$commute with the $z$ component of the total angular momentum operator $S_{z}$ of the $N$ spins. This property directly implies that the states $A_{+}|r, r, \nu\rangle$ and $A_{-}|r,-r, v\rangle$ are eigenstates of $S_{z}$ with eigenvalues $r$ and $-r$, respectively. We have, indeed,

$$
\begin{aligned}
S_{z} A_{ \pm}|r, \pm r, v\rangle & =A_{ \pm} A_{ \pm}^{-1} S_{z} A_{ \pm}|r, \pm r, v\rangle \equiv A_{ \pm} S_{z}|r, \pm r, v\rangle \\
& = \pm r A_{ \pm}|r, \pm r, v\rangle .
\end{aligned}
$$

On the other hand, it is easy to check that they are also eigenstates of $H_{0}$ corresponding to the eigenvalues $\left(\omega_{0}+2 r \omega\right)$ and $-\left(\omega_{0}+2 r \omega\right)$, respectively. This circumstance in turn means that these states are also eigenstates of the total Hamiltonian given by Eq. (1), simultaneously being eigenstates of $H_{0}$ and $H_{I}$. In other words the IFE states space may be represented as a direct sum of appropriate vectorial subspaces invariant under the action of the total Hamiltonian $H$. As a consequence we might envision initial conditions from which the system effectively evolves while conserving the value of its initial entanglement no matter the measure used. Our results on the structure of $\mathcal{N}_{0}$ play an important role in the context of the problem of the diagonalization of the nonhomogeneous spin-star-system Hamiltonian model under scrutiny in this section. Recently, indeed, many efforts have been made in order to find the spectrum of such a Hamiltonian, but, until now, only a particular set of eigensolutions has been known [42]. 


\section{CONCLUDING REMARKS}

In this paper we have introduced a class of states of a bipartite system christened IFE states. This set of states encompasses all those initial conditions of the compound system from which each subsystem evolves with no energy exchange with the other one and leaving the level of mutual entanglement unmodified regardless of which measure is adopted. These properties stem from cooperative effects leading, through quantum interference processes, to the cancellation of any dynamical consequence of the coupling term $H_{I}$. We stress that since the constructions of the IFE states space require resolving their characteristic equations in the Hilbert state of the given bipartite system, it may be empty. It is, however, worth noticing that when subradiant states exist, they are IFE states too, allowing us to claim that our definition of IFE states generalizes, indeed, that of the subradiant state. Our main result is constituted by the two characteristic equations of the states (Theorem 1 and Corollary 3 ) as well as the construction of the set of all IFE states of a nontrivial Hamiltonian model of evergreen interest. A remarkable merit of such a result is its universality with respect to time-independent Hamiltonian models, which means that the characteristic equations reported here are applicable to any bipartite system evolving unitarily. The concept of IFE states is illustrated by a nonhomogeneous spin-star system. We were able to find IFE states belonging to $\mathcal{N}_{0}$. It would be interesting to develop a physical example possessing IFE states outside the set $\mathcal{N}_{0}$, i.e., to go beyond the standard dark states. The more intriguing situation corresponding to the evolution of a bipartite system in the presence of an environment is currently under investigation and will be presented elsewhere.

\section{ACKNOWLEDGMENTS}

The authors thank the anonymous referee for valuable remarks. D.C. was partially supported by the National Science Center project DEC-2011/03/B/ST2/00136.

\section{APPENDIX}

Taking the time derivative of Eq. (3), one obtains

$$
H e^{-i H t}|\psi\rangle=\left[\dot{\alpha}(t)+H_{0}\right] e^{-i \alpha(t)} e^{-i H_{0} t}|\psi\rangle,
$$

and thus

$$
H e^{-i H t}|\psi\rangle=\left[\dot{\alpha}(t)+H_{0}\right] e^{-i H t}|\psi\rangle .
$$

Now, due to $H=H_{0}+H_{I}$ the above equation reduces to

$$
H_{I} e^{-i H t}|\psi\rangle=\dot{\alpha}(t) e^{-i H t}|\psi\rangle,
$$

which leads to

$$
\left\langle\psi\left|e^{i H t} H_{I} e^{-i H t}\right| \psi\right\rangle=\dot{\alpha}(t)\left\langle\psi\left|e^{i H t} e^{-i H t}\right| \psi\right\rangle=\dot{\alpha}(t) .
$$

Note, however, that if $|\psi\rangle$ is an IFE state, the quantity $\left\langle\psi\left|e^{i H t} H_{I} e^{-i H t}\right| \psi\right\rangle$ has to be time independent. Indeed, using the fact that $\left\langle\psi\left|e^{i H t} H e^{-i H t}\right| \psi\right\rangle=\langle\psi|H| \psi\rangle$ is time independent, one has

$$
\begin{aligned}
& \left\langle\psi\left|e^{i H t} H e^{-i H t}\right| \psi\right\rangle \\
& =\left\langle\psi\left|e^{i H t} H_{0} e^{-i H t}\right| \psi\right\rangle+\left\langle\psi\left|e^{i H t} H_{I} e^{-i H t}\right| \psi\right\rangle \\
& =\left\langle\psi\left|H_{0}\right| \psi\right\rangle+\left\langle\psi\left|e^{i H t} H_{I} e^{-i H t}\right| \psi\right\rangle,
\end{aligned}
$$

which proves that $\alpha(t)=\alpha_{0}+\alpha t$.
[1] R. H. Dicke, Phys. Rev. 93, 99 (1954).

[2] M. Gross and S. Haroche, Phys. Rep. 93, 301 (1982).

[3] D. Petrosyan and G. Kurizki, Phys. Rev. Lett. 89, 207902 (2002).

[4] A. Kalachev, Phys. Rev. A 76, 043812 (2007).

[5] R. Ben-Av and I. Exman, Phys. Rev A 84, 014301 (2011).

[6] S. Strauf, K. Hennessy, M. T. Rakher, Y.-S. Choi, A. Badolato, L. C. Andreani, E. L. Hu, P. M. Petroff, and D. Bouwmeester, Phys. Rev. Lett. 96, 127404 (2006).

[7] L. Di Carlo, J. M. Chow, J. M. Gambetta, L. S. Bishop, B. R. Johnson, D. I. Schuster, J. Majer, A. Blais, L. Frunzio, S. M. Girvin, and R. J. Schoelkopf, Nature (London) 460, 240 (2009).

[8] D. Ferrari, G. L. Celardo, G. P. Berman, R. T. Sayre, and F. Borgonovi, J. Phys. Chem. C 118, 20 (2014).

[9] A. Sitek and A. Manolescu, Phys. Rev. A 88, 043807 (2013).

[10] G. S. Agarwal, in Quantum Statistical Theories of Spontaneous Emission and Their Relation to Other Approaches, edited by G. Hhler, Springer Tracts in Modern Physics Vol. 70 (Springer, Berlin, 1974).

[11] A. Auffeves, D. Gerace, S. Portolan, A. Drezet, and M. Franca Santos, New J. Phys. 13, 093020 (2011).

[12] V. N. Pustovit, A. M. Urbas, and T. V. Shahbazyan, Phys. Rev. B 88, 245427 (2013).

[13] V. N. Pustovit and T. V. Shahbazyan, Phys. Rev. Lett. 102, 077401 (2009).
[14] V. N. Pustovit and T. V. Shahbazyan, Phys. Rev. B 82, 075429 (2010).

[15] T. Bienaimè, N. Piovella, and R. Kaiser, Phys. Rev. Lett. 108, 123602 (2012).

[16] P. Lodahl, A. F. van Driel, I. S. Nikolaev, A. Irman, K. Overgaag, D. Vanmaekelbergh, and W. L. Vos, Nature (London) 430, 654 (2004).

[17] R. Wiegner, J. von Zanthier, and G. S. Agarwal, Phys. Rev. A 84, 023805 (2011).

[18] G. Benivegna and A. Messina, J. Mod. Opt. 36, 1205 (1989).

[19] G. Benivegna and A. Messina, Phys. Lett. A 126, 249 (1988).

[20] S. Nicolosi, A. Napoli, A. Messina, and F. Petruccione, Phys. Rev. A 70, 022511 (2004).

[21] S. Nicolosi, A. Napoli, and A. Messina, Eur. Phys. J. D 33, 113 (2005).

[22] A. Crubellier, S. Liberman, D. Pavolini, and P. Pillet, J. Phys. B: At. Mol. Phys. 18, 3811 (1985).

[23] A. Crubellier and D. Pavolini, J. Phys. B: At. Mol. Phys. 19, 2109 (1986).

[24] A. Crubellier, J. Phys. B: At. Mol. Phys. 20, 971 (1987).

[25] A. Crubellier and D. Pavolini, J. Phys. B: At. Mol. Phys. 20, 1451 (1987).

[26] D. Petrosyan and G. Kurizki, Quantum Inf. Comput. 6, 001 (2006) 
[27] D. Pavolini, A. Crubellier, P. Pillet, L. Cabaret, and S. Liberman, Phys. Rev. Lett. 54, 1917 (1985).

[28] M. Scheibner, T. Schmidt, L. Worschech, A. Forchel, G. Bacher, T. Passow, and D. Hommel, Nat. Phys. 3, 106 (2007).

[29] K. Baumann, Ch. Guerlin, F. Brennecke, and T. Esslinger, Nature (London) 464, 1301 (2010).

[30] S. Filipp, A. F. van Loo, M. Baur, L. Steffen, and A. Wallraff, Phys. Rev. A 84, 061805(R) (2011).

[31] Y. Takasu, Y. Saito, Y. Takahashi, M. Borkowski, R. Ciurylo, and P. S. Julienne, Phys. Rev. Lett. 108, 173002 (2012).

[32] R. G. DeVoe and R. G. Brewer, Phys. Rev. Lett. 76, 2049 (1996).

[33] P. Zanardi and M. Rasetti, Phys. Rev. Lett. 79, 3306 (1997).

[34] P. Zanardi and F. Rossi, Phys. Rev. Lett. 81, 4752 (1998).
[35] A. Kalachev and S. Kroll, Phys. Rev. A 74, 023814 (2006).

[36] G. Benivegna and A. Messina, Europhys. Lett. 10, 243 (1989).

[37] S. Ashhab, J. R. Johansson, A. M. Zagoskin, and F. Nori, Phys. Rev. A 75, 063414 (2007).

[38] A. Lidar and K. B. Whaley, in Irreversible Quantum Dynamics, edited by F. Benatti and R. Floreanini, Springer Lecture Notes in Physics Vol. 622 (Springer-Verlag, Berlin, Heidelberg, 2003), pp. 83-120.

[39] D. A. Lidar, Adv. Chem. Phys. 154, 295 (2014).

[40] P. Zanardi and M. Rasetti, Mod. Phys. Lett. B 11, 1085 (1997).

[41] The authors thank the referee for this suggestion.

[42] M. A. Jivulescu, E. Ferraro, A. Napoli, and A. Messina, Rep. Math. Phys. 64, 315 (2009). 